\title{
Increasing the density melts ultrasoft colloidal glasses
}

\author{
Ludovic Berthier, ${ }^{1}$ Angel J. Moreno, ${ }^{2,3}$ and Grzegorz Szamel ${ }^{4}$ \\ ${ }^{1}$ Laboratoire des Collö̈des, Verres et Nanomatériaux, UMR CNRS 5587, Université Montpellier 2, 34095 Montpellier, France \\ ${ }^{2}$ Centro de Física de Materials, CSIC, UPV/EHU, Paseo Manuel de Lardizabal 5, E-20018 San Sebastián, Spain \\ ${ }^{3}$ Materials Physics Center MPC, Paseo Manuel de Lardizabal 5, E-20018 San Sebastián, Spain \\ ${ }^{4}$ Department of Chemistry, Colorado State University, Fort Collins, Colorado 80523, USA
}

(Received 23 September 2010; revised manuscript received 22 October 2010; published 14 December 2010)

\begin{abstract}
We use theory and simulations to investigate the existence of amorphous glassy states in ultrasoft colloids. We combine the hypernetted chain approximation with mode-coupling theory to study the dynamic phase diagram of soft repulsive spheres interacting with a Hertzian potential, focusing on low temperatures and large densities. At constant temperature, we find that an amorphous glassy state is entered upon compression, as in colloidal hard spheres, but the glass unexpectedly melts when density increases further. We attribute this reentrant fluid-glass transition to particle softness and correlate this behavior to previously reported anomalies in soft systems, thus emphasizing its generality. The predicted fluid-glass-fluid sequence is confirmed numerically.
\end{abstract}

DOI: 10.1103/PhysRevE.82.060501

PACS number(s): 64.70.pv, 05.20.Jj, 64.70.qd

The hard-sphere system is an emblematic model in statistical physics. It is a useful theoretical model for liquids which can be studied experimentally using colloids [1]; its density driven fluid-crystal and fluid-glass transitions have been extensively studied. Recently, there has been growing interest in systems composed of ultrasoft particles, which can be realized experimentally using colloidal particles made of star-shaped polymers or microgels [2]. Since softness introduces (at least) one more dimension to the phase diagram, new physical behavior can be expected from the competition between entropy, which governs the behavior of hard spheres, and energy, which appears when soft particles overlap.

As a result, the physics of systems consisting of soft particles is considerably richer than that of hard ones. Unusual equilibrium phase diagrams arise depending on the particular features of particle softness. These include cluster crystal structures at arbitrarily large densities [3] and a nonmonotonic density dependence of the freezing transition [4-8] with a complex cascade of crystalline states [9]. In the latter case, the thermodynamically stable fluid phase also exhibits density anomalies at the structural [7-10] and dynamical $[9,11,12]$ levels.

Exploring the existence of amorphous solid states at low temperature and large density in soft particle materials is an emerging field. The glassy dynamics of star polymers have been investigated both theoretically and experimentally, and, in particular, nontrivial phase diagrams and novel glassy states have been described in star-polymer mixtures $[2,13,14]$. Several studies were dedicated to the perturbations brought about by adding an energy scale to the hard-sphere glassy behavior. Such a perturbation not only shifts the glass transition to larger densities $[15,16]$ but also affects qualitatively the dynamics near the transition, in particular the glass fragility $[15,18,19]$. Experiments have also investigated the physics of glass aging [20], mostly in microgels, with a particular interest on the jamming transition intervening deep into the glass phase [21,22].

In this work we investigate whether the anomalous equilibrium phase diagram and transport properties of soft repul- sive sphere systems also impact their transition to a nonergodic glassy state. This possibility was evoked for star polymers [13] for a narrow range of star functionalities but was not confirmed numerically because crystallization intervened before slow dynamics set in. Other theoretical studies of soft repulsive spheres [16] focused on densities close to the hard-sphere transition, leaving the behavior at large densities unexplored [17].

Our main finding is that an "anomalous" density dependence of the glass transition temperature is found both theoretically and in computer simulations for the well-studied Hertzian model for ultrasoft particles. Thus, it is possible to melt a glass made of soft particles by increasing its density, a scenario that has not been observed yet in experiments. This is surprising on general grounds because particle crowding typically yields jammed, rather than fluid, states for "usual" particles such as hard spheres or molecular fluids. Indeed, traffic jams are rarely eased by putting more cars on the road.

We consider $N$ particles in a volume $V$ interacting with a soft, repulsive, pairwise additive Hertzian potential,

$$
V(r<\sigma)=\epsilon(1-r / \sigma)^{5 / 2}
$$

and $V(r \geq \sigma)=0$. Originally derived to describe the elastic repulsion between deformable spheres, the Hertzian potential has recently been used to study theoretically the physics of soft repulsive particles in various contexts and seems to describe well the interaction between microgel colloids [21]. Its physics is very similar to the harmonic sphere model inspired by the physics of foams [23] or to the Gaussian core model [4] derived from polymer physics. The control parameters of the system are the particle number density, $\rho=N \sigma^{3} / V$, and the ratio of the temperature $T$ and energy scale $\epsilon$. Since particles can overlap at a finite-energy cost, $\rho$ can significantly exceed 1 and, indeed, we are interested in this high-density regime. We use reduced units, measuring lengths in units of $\sigma$, and temperatures in units of $\epsilon$.

We study the structure and dynamics of the Hertzian spheres combining theory and simulations. For the theory, we closely follow an earlier investigation of harmonic 

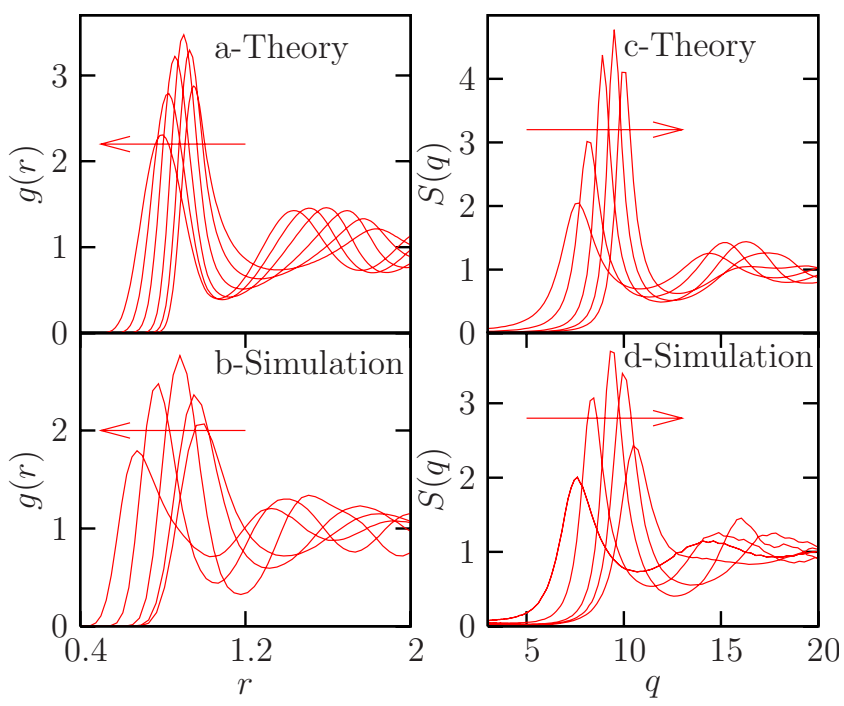

FIG. 1. (Color online) Nonmonotonic evolution of structural quantities with increasing density (arrows) as predicted by theory (top) and obtained from MD simulations (bottom). (a) Predicted pair-correlation function for $T=0.00287$ and $\rho=1.1,1.4,1.7,2.1$, 2.43, and 2.84; (b) numerically measured $g(r)$ for $T=0.0043$ and $\rho=1.2,1.45,2.05,3.0$, and 4.05 ; (c) predicted structure factor for $T=0.00287$ and $\rho=1.1,1.5,2.1,2.63$, and 2.84; and (d) numerically measured $S(q)$ for $T=0.0043$ and $\rho=1.2,1.7,2.4,3.0$, and 4.05 .

spheres to which we refer for further details and equations [16]. We examine a monodisperse system of the Hertzian spheres using the hypernetted chain (HNC) approximation [24] to obtain the pair-correlation function $g(r)$ and the structure factor $S(q)$ for a given state point $(\rho, T)$. We then inject $S(q)$ in the dynamical equations of motion for timecorrelation functions derived within mode-coupling theory (MCT) [25], which we solve numerically [26]. We obtain the time dependence of density-density correlation functions for a wide range of wave vectors for a broad range of densities and temperatures.

To verify theoretical predictions we perform moleculardynamics (MD) simulations of a polydisperse Hertzian sphere system. Polydispersity is needed to prevent crystallization or fractionation that easily occurs in the monodisperse system. The particle diameters $\sigma_{i}$ are randomly drawn from a flat probability distribution with $0.826 \leq \sigma_{i} \leq 1.126$. Two particles $i, j$ interact through Eq. (1) with $\sigma=\left(\sigma_{i}+\sigma_{j}\right) / 2$. We use $N=9000$ and periodic boundary conditions. Equilibration is achieved through periodic velocity rescaling; production runs are carried out in the microcanonical ensemble.

We now discuss two representative quantities characterizing the structure of the fluid at various state points. In Fig. 1(a), we show the evolution with the density of the pair-correlation function $g(r)$ at constant temperature, $T=0.00287$, as predicted by the HNC approximation. As expected, with increasing density particles are getting closer to each other and as a result, the position of the first peak of $g(r)$ moves toward smaller values of $r$. The monotonic shift of the peak position of $g(r)$ is accompanied by an anomalous nonmonotonic evolution of the peak height [9]. Specifically, with increasing density from $\rho=1.1$ to 1.7 the height of the first peak increases, but it decreases when increasing density further from $\rho=1.7$ to 2.84. This evolution is anomalous in the sense that the first peak of $g(r)$ typically increases monotonically with density in simple liquids at constant temperature. The locus of the density anomaly evolves with temperature, as studied in detail for the harmonic potential [10].

Since $g(r)$ and the structure factor $S(q)$ are related via the Fourier transform [24], it is not surprising that the same anomalous trend is observed in Fig. 1(c), which shows the HNC-derived $S(q)$ for the same temperature. With increasing density the position of the first peak shifts to larger $q$, corresponding to smaller interparticle distances. Simultaneously, the peak height exhibits a nonmonotonic variation. The maxima of $g(r)$ and $S(q)$ typically occur at slightly different densities, $\rho \approx 1.7$ and $\rho \approx 2.6$, respectively, at $T=0.00287$.

The trends exhibited by $g(r)$ and $S(q)$ calculated using the HNC approximation are also evident in the simulations. In Figs. 1(b) and 1(d) we show the pair-correlation function and the structure factor obtained in simulations at constant $T=0.0043$ and various densities. Both quantities exhibit a nonmonotonic evolution with density, $g(r)$ having its maximum near $\rho \approx 2.05$ and $S(q)$ near $\rho \approx 2.4$. Thus, the two maxima are closer to each other in simulations than according to theory. However, the similarity between theory and simulations is quite striking from these plots, showing that HNC is performing reasonably well for the explored state points. Quantitative agreement cannot be expected as we are using different particle size distributions in both approaches. In particular, we believe that polydispersity is responsible for the fact that the first peaks of both $g(r)$ and $S(q)$ obtained from MD are quite a bit smaller and broader than those calculated using the HNC approximation.

The nonmonotonic dependence of the height of the first peak of $g(r)$ on density was previously discussed for several similar models. Physically, it originates from a competition between energy and entropy with an anomalous outcome due to the extreme softness of the particles [7-10]. Briefly, at smaller densities, the system can easily minimize its free energy by avoiding the energetically costly particle overlaps. In this regime, the system effectively behaves as hard spheres, with $g(r)$ and $S(q)$ becoming sharper as $\rho$ increases. At larger densities, it becomes difficult, and thus entropically costly, for the system to find configurations with no overlap (note that the nominal volume fraction $\phi=\pi \rho / 6$ may exceed $100 \%)$. Rather than trying to do the impossible, particles increase their overlaps, have effectively more space, and may thus access a larger number of configurations. This increasing disorder leads to the broadening of the first peak of correlation functions. The entropy gain can be larger than the energy cost of the overlaps because the Hertzian potential is bounded at any separation.

The above described competition between entropic and energetic effects is purely statistical. It is reasonable to expect that the resulting structural anomalies will cause a nontrivial density dependence of the equilibrium freezing transition [9]. However, this competition and the anomalies are not obviously relevant to the nonequilibrium glass transition, which is related to the increasing difficulty to transport matter with increasing particle crowding. Our main result is that the anomalous density dependence of equilibrium structural 

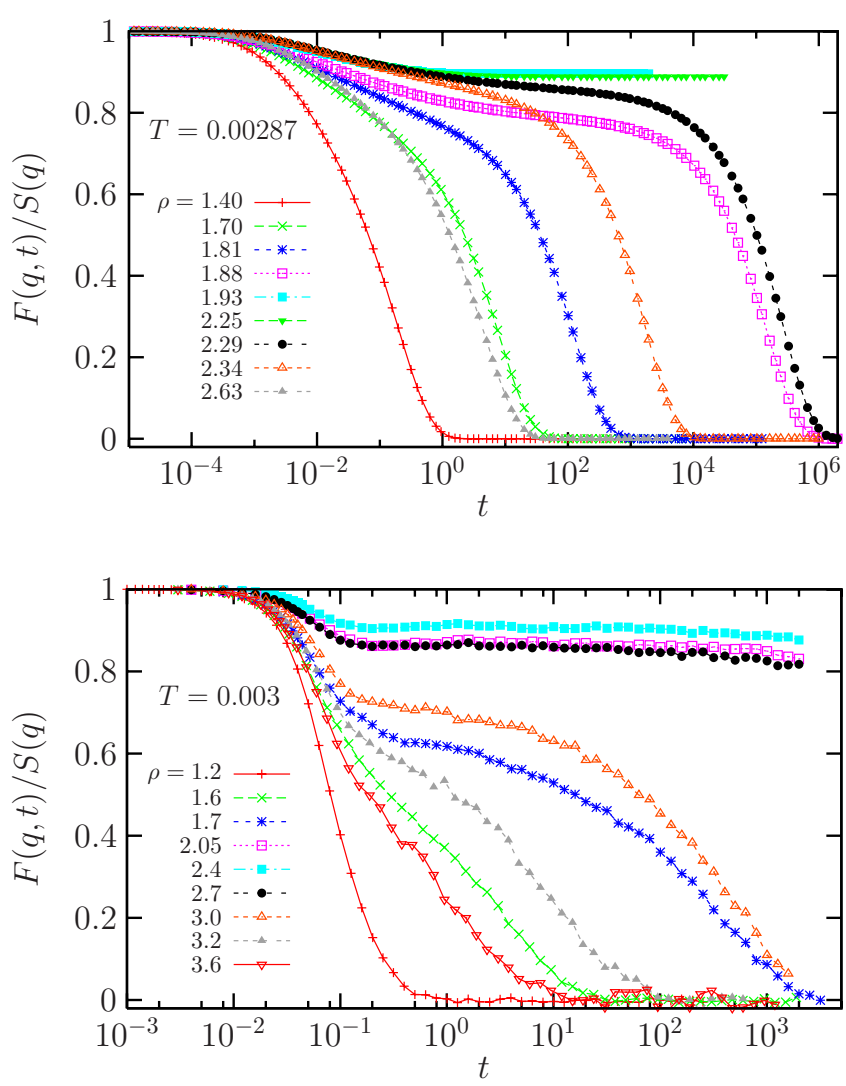

FIG. 2. (Color online) Nonmonotonic evolution of timecorrelation functions with density at constant temperature for theory (top, $q=9.1$ ) and simulations (bottom, $q=9.4$ ). A two-step decay initially develops when $\rho$ increases, nonergodic glass states are obtained at intermediate densities, and the glass melts upon further compression.

quantities is indeed accompanied by the nonmonotonic dependence of structural relaxation and ergodicity breaking. Anomalous density dependence of transport properties was found before in the thermodynamically stable nonglassy soft particle fluid [7,9,11-13] but remained essentially unexplored for the deeply supercooled state and for the glass.

In Fig. 2 we show the $\rho$ dependence of the density-density time correlators as obtained from both theory and simulations. As for structural quantities we show the correlators at constant $T$ and increasing $\rho$, which expose the most remarkable features of the data, but a much broader range of state points was explored. Both panels of Fig. 2 exhibit the same qualitative behavior. With increasing density the relaxation becomes progressively slower and the fluid approaches a glass transition qualitatively similar to the one observed with hard particles. A two-step decay of time-correlation functions emerges, with the slow relaxation being strongly dependent on density. For the temperatures shown in Fig. 2, $F(q, t)$ does not decay to zero above $\rho \approx 1.9$ within MCT at $T=0.00287$ and above $\rho \approx 2$ within MD at $T=0.003$. Although the relaxation time is infinite within theory, we can simply report that it has become too large to be measured in the simulations and that, for practical purposes, the system is a nonergodic glass. In the glass phase there is, at first, little change when $\rho$ increases, apart from a weak variation of the long-time limit

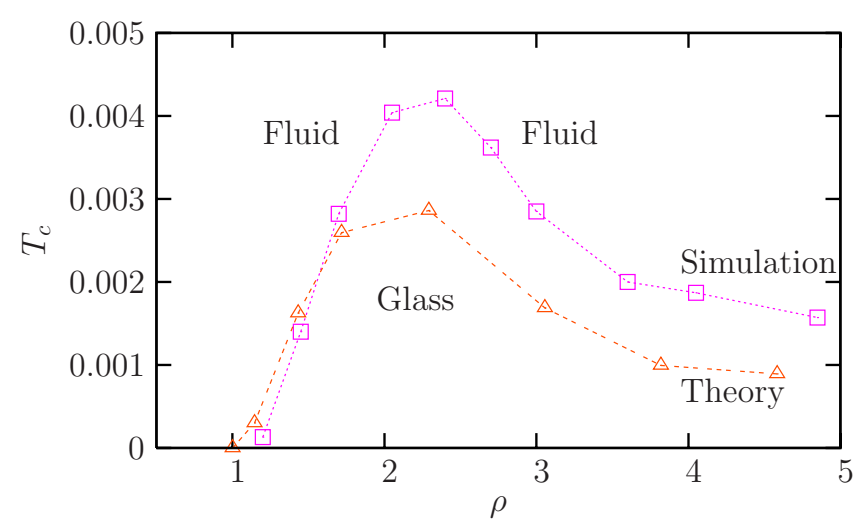

FIG. 3. (Color online) Dynamic phase diagram showing the exact (theory) and fitted (simulations) critical temperatures obtained from power law fits to the structural relaxation times. A reentrant glass transition is obtained in both cases with a maximum near $\rho \approx 2.4$.

of the correlators. However, quite surprisingly, the glass suddenly melts when it is compressed further, and the relaxation time becomes finite again. This is counterintuitive from the standpoint of glass physics, although perhaps not totally unexpected for a system known to displaying other anomalies. Past the glass melting transition, the dynamics becomes faster when density is increased even further.

The above description of time-dependent correlators suggests the existence of a reentrant glass transition line in the $(\rho, T)$ phase diagram, which we confirm in Fig. 3 . In the theory we easily obtain the critical mode-coupling temperature, $T_{c}(\rho)$, with arbitrary precision, by fitting the temperature evolution at constant density of the structural relaxation time to a power-law divergence, $\tau \propto\left(T-T_{c}\right)^{-\gamma}$ [25]. A finite mode-coupling temperature emerges in a way qualitatively similar to the results obtained for the harmonic potential [16]. However, the mode-coupling transition line has a clear maximum, $T_{c} \approx 0.003$, near $\rho \approx 2.4$, slightly below the density where $S(q)$ has its maximum. It then decreases smoothly with increasing $\rho$. When performed for simulation data, the same analysis works well over a moderate range of two to three decades of $\tau$ but, as usual [25], the MCT power law breaks down below some $T$. Remarkably, however, the numerical $T_{c}$ values reported in Fig. 3 exhibit trends in good qualitative agreement with theory. The important point is the robustness of the reentrant glass transition line with a maximum around $\rho \approx 2.4$ at nearly the same density where the freezing transition temperature peaks for the monodisperse system [9], $T_{m} \approx 0.008$.

The quantitative disagreement between the two glass lines has multiple origins. First, the theoretical results were obtained for the monodisperse system, but the simulations were performed using a polydisperse one. Second, both HNC and MCT have their well-known limitations. It is possible to improve the static approximation [24], but the predicted (spurious) divergence of the relaxation time predicted by MCT will remain. Moreover, MCT predictions stem from the knowledge of pair-correlation functions, incorrectly ignoring additional structural information [27]. Nevertheless, in the present case, the evolution of pair correlations is certainly the 
major origin of the reentrant glass transition, which is thus successfully captured by MCT. Analogous successes of MCT occurred in the past, for instance, for sticky colloidal particles [28]. Despite its limitations, our procedure should therefore be useful in revealing new dynamical features relevant in the context of colloidal physics with soft interactions, as our numerical simulations confirm.

In summary, by combining simple analytical approaches and simulations, we predicted and observed numerically the existence of a reentrant fluid-glass transition in a system of Hertzian spheres at large densities. We believe that our results are not restricted to this particular interaction and simi- lar behavior should arise in other systems with soft repulsive potentials and should find experimental realizations using ultrasoft colloids. Our results suggest that making softer colloids not only affects the kinetic glass fragility $[15,18,19]$ but also produces nonintuitive effects such as a reentrant glass transition, which remains to be seen in an experiment.

We acknowledge financial support from ANR Dynhet and Région Languedoc-Roussillon (L.B.), projects MAT200763681 (MEC, Spain) and IT-436-07 (Basque Government, Spain) (A.J.M.), and NSF Grant No. CHE 0909676 (G.S.).
[1] P. N. Pusey and W. van Megen, Nature (London) 320, 340 (1986).

[2] C. N. Likos, Soft Matter 2, 478 (2006).

[3] B. M. Mladek, P. Charbonneau, C. N. Likos, D. Frenkel, and G. Kahl, J. Phys.: Condens. Matter 20, 494245 (2008).

[4] F. H. Stillinger, J. Chem. Phys. 65, 3968 (1976).

[5] T. A. Witten, P. A. Pincus, and M. E. Cates, Europhys. Lett. 2, 137 (1986).

[6] M. Watzlawek, H. Löwen, and C. Likos, J. Phys.: Condens. Matter 10, 8189 (1998).

[7] A. Lang, C. N. Likos, M. Watzlawek, and H. Löwen, J. Phys.: Condens. Matter 12, 5087 (2000).

[8] A. A. Louis, P. G. Bolhuis, and J. P. Hansen, Phys. Rev. E 62, 7961 (2000).

[9] J. C. Pàmies, A. Cacciuto, and D. Frenkel, J. Chem. Phys. 131, 044514 (2009).

[10] H. Jacquin and L. Berthier, Soft Matter 6, 2970 (2010).

[11] W. P. Krekelberg, T. Kumar, J. Mittal, J. R. Errington, and T. M. Truskett, Phys. Rev. E 79, 031203 (2009).

[12] S. A. Egorov, J. Chem. Phys. 128, 174503 (2008).

[13] G. Foffi, F. Sciortino, P. Tartaglia, E. Zaccarelli, F. Lo Verso, L. Reatto, K. A. Dawson, and C. N. Likos, Phys. Rev. Lett. 90, 238301 (2003).

[14] C. Mayer, E. Zaccarelli, E. Stiakakis, C. N. Likos, F. Sciortino, A. Munam, M. Gauthier, N. Hadjichristidis, H. Iatrou, P. Tartaglia, H. Löwen, and D. Vlassopoulos, Nature Mater. 7, 780 (2008).

[15] L. Berthier and T. A. Witten, EPL 86, 10001 (2009); Phys.
Rev. E 80, 021502 (2009).

[16] L. Berthier, E. Flenner, H. Jacquin, and G. Szamel, Phys. Rev. E 81, 031505 (2010).

[17] See, however, A. Ikeda and K. Miyazaki, e-print arXiv:1008.2597.

[18] J. Mattsson, H. M. Wyss, A. Fernandez-Nieves, K. Miyazaki, Z. Hu, D. R. Reichman, and D. A. Weitz, Nature (London) 462, 83 (2009).

[19] J. Yang and K. S. Schweizer, EPL 90, 66001 (2010).

[20] P. Yunker, Z. Zhang, K. B. Aptowicz, and A. G. Yodh, Phys. Rev. Lett. 103, 115701 (2009).

[21] Z. Zhang, N. Xu, D. T. N. Chen, P. Yunker, A. M. Alsayed, K. B. Aptowicz, P. Habdas, A. J. Liu, S. R. Nagel, and A. G. Yodh, Nature (London) 459, 230 (2009).

[22] D. A. Sessoms, I. Bischofberger, L. Cipelletti, and V. Trappe, Philos. Trans. R. Soc. London, Ser. A 367, 5013 (2009).

[23] D. J. Durian, Phys. Rev. Lett. 75, 4780 (1995).

[24] J. P. Hansen and I. R. McDonald, Theory of Simple Liquids (Elsevier, Amsterdam, 1986).

[25] W. Götze, Complex Dynamics of Glass-Forming Liquids: A Mode-Coupling Theory (Oxford University Press, Oxford, 2008).

[26] E. Flenner and G. Szamel, Phys. Rev. E 72, 031508 (2005).

[27] L. Berthier and G. Tarjus, Phys. Rev. Lett. 103, 170601 (2009); Phys. Rev. E 82, 031502 (2010).

[28] K. N. Pham, A. M. Puertas, J. Bergenholtz, S. U. Egelhaaf, A. Moussaid, P. N. Pusey, A. B. Schofield, M. E. Cates, M. Fuchs, and W. C. K. Poon, Science 296, 104 (2002). 